\title{
Philosophiques
}

\section{Mario Bunge, Chasing Reality: Strife over Realism,Toronto, University of Toronto Press (coll. Toronto Studies in Philosophy), 2006, 342 p.}

\section{Davy Mougenot}

Volume 35, numéro 2, automne 2008

URI : https://id.erudit.org/iderudit/000445ar

DOI : https://doi.org/10.7202/000445ar

Aller au sommaire du numéro

Éditeur(s)

Société de philosophie du Québec

ISSN

0316-2923 (imprimé)

1492-1391 (numérique)

Découvrir la revue

Citer ce compte rendu

Mougenot, D. (2008). Compte rendu de [Mario Bunge, Chasing Reality: Strife over Realism,Toronto, University of Toronto Press (coll. Toronto Studies in

Philosophy), 2006, 342 p.] Philosophiques, 35(2), 609-612.

https://doi.org/10.7202/000445ar d'utilisation que vous pouvez consulter en ligne.

https://apropos.erudit.org/fr/usagers/politique-dutilisation/ 


\title{
Comptes rendus
}

\author{
Mario Bunge, Chasing Reality: Strife over Realism, Toronto, University \\ of Toronto Press (coll. Toronto Studies in Philosophy), 2006, 342 p.
}

Le livre Chasing Reality (2007), de Mario Bunge, se présente comme la défense d'un réalisme scientifique qui prend l'envergure d'un véritable système du monde (à peu près tous les sujets imaginables y sont traités) et d'un pamphlet contre ceux qui s'y opposent (tous ceux qui soutiennent une forme d'idéalisme ou de phénoménisme, les anti-réalistes et les socio-constructivistes, essentiellement).

L'expression «système du monde « rappelle l'œuvre du baron d'Holbach, dont Bunge revendique la filiation, et trace une méthodologie philosophique à la fois simple et terriblement ambitieuse. En effet, dans son ouvrage, Bunge reconstruit littéralement le monde, de ses éléments les plus « simples » aux éléments les plus complexes: il pose d'abord les bases ontologiques de son système (réalisme matérialiste ou hyloréalisme), expose ensuite l'épistémologie qui s'y rapporte (scientisme: les sciences constituent notre meilleur moyen d'établir des connaissances), nous montre comment son système permet d'affronter efficacement les principaux problèmes intellectuels de divers domaines (évidemment en philosophie, mais la biologie, la médecine, la psychologie et les sciences sociales sont abordées). Finalement, Bunge envisage même une extension du système à la morale (quelques remarques sont également faites dans la sphère politique).

Le livre se divise donc classiquement: les quatre premiers chapitres couvrent l'ontologie et la métaphysique, les cinq suivants l'épistémologie et la philosophie des sciences avec, en guise de conclusion, une ouverture sur le réalisme moral. Notre compte rendu ne sera donc pas structuré de façon plus originale et suivra les thèses essentielles de l'auteur (nous n'aborderons pas, cependant, les thèses morales qui constituent une partie très restreinte du livre).

D’entrée de jeu, disons abruptement que Bunge défend un réalisme matérialiste (émergentiste), qu'il nomme hyloréalisme, selon lequel la réalité est constituée de choses matérielles que l'on peut connaître par l'entremise cognitive des sciences, plus particulièrement par la découverte des mécanismes qui sous-tendent les divers phénomènes que nous cherchons à comprendre. Autrement dit, Bunge propose de s'aventurer dans la triade fait-apparence-fiction, les faits constituant ce qui se produit indépendamment de l'esprit, les apparences étant les faits tels qu'ils nous apparaissent (phénomènes) et les fictions étant les constructions mentales chargées de rendre intelligible le monde et les phénomènes.

Afin d'éclaircir ce que Bunge entend par fait et de défendre le réalisme, c'est-àdire la thèse selon laquelle le monde existe indépendamment de l'esprit, il faut partir de la notion plus fondamentale de "chose » : L'affirmation selon laquelle le monde est l'ensemble des choses est difficilement contestable» (p. 10). Assurément, les faits (le chat est sur le tapis) impliquent des choses (chat, tapis). Bunge s'oppose à la caractérisation cartésienne de "chose concrète » en tant que res extensa (puisqu'elle la limite aux choses solides, et les choses solides ne sont qu'une infime partie de notre univers), pour épouser celle de Platon : «Je propose que la mutabilité soit la propriété partagée par toutes les choses concrètes [...]» (p. 10). De plus, et, techniquement, la mutabilité 


\section{Philosophiques / Automne 2008}

est la propriété universelle conférée par l'énergie. Une chose est donc tout ce qui possède de l'énergie (sous toutes ses formes: mécanique, gravitationnelle, nucléaire, etc.) et est donc muable. Toutefois, cette caractérisation universaliste ne permet pas de différencier une chose d'une chose concrète et, pour cela, nous devons avoir recours aux propriétés saillantes de cette chose particulière ainsi qu'à la nature des changements qu'elles peuvent subir. Les propriétés des choses se divisent en plusieurs couples : 1) essentiel/accidentel ; 2) fondamental/dérivé ; 3) première/seconde ; 4) invariant/relatif. Les changements peuvent être quantitatifs (ils n'affectent pas la nature de la propriété) ou qualitatifs (les changements affectent la nature de la propriété). Le phénomène des changements qualitatifs est philosophiquement (et factuellement) capital, car en lui repose la possibilité d'expliquer matériellement les phénomènes d'émergence qui nous intéressent tant (la vie et l'esprit).

Définir une chose concrète revient à reconnaître qu'elle est un $x$ muable (parce qu'énergétique) possédant un certain nombre de propriétés qu'il s'agit de décrire pour lui conférer son identité. Dans l'hyloréalisme, un fait est une chose concrète dans un certain état (fait statique) ou quand elle s'achemine vers un état (fait dynamique). Une autre distinction importante au sujet des faits, reprise de Searle, porte sur les faits «bruts" et «institutionnels": les premiers sont ceux qui existent naturellement, indépendamment du fait d'avoir été initialement pensés par un esprit humain (les tremblements de terre) alors que les seconds sont artefactuels ou artificiels dans le sens où ils dépendent de l'esprit humain (la monnaie).

Cette rapide excursion dans une ontologie réaliste est logiquement enchaînée avec l'éternelle question philosophique du statut des phénomènes et de notre capacité à connaître au-delà de ceux-ci ( «l'hypothétique véritable monde»). Bunge expose donc, dans son deuxième et troisième chapitre, une histoire condensée de la lutte entre phénoménalisme/empirisme/idéalisme et le réalisme (essentiellement, le mécanisme scientifico-philosophique). Bunge distingue les deux camps en affirmant que les phénoménalistes/empiristes/idéalistes tentent de comprendre le monde des apparences à l'aide des qualités secondes (de ce que nous apprend les sens ou l'esprit); alors que les mécanistes/réalistes tentent de comprendre le monde et les apparences à l'aide des qualités premières et secondes (Locke reçoit cependant l'attention qu'il mérite, étant un réaliste tout en disqualifiant la possibilité de connaître de façon universelle et certaine au-delà de nos sens). L'époque moderne est décrite comme un affrontement entre ces deux courants, Berkeley et Hume représentant la «contre-révolution » du mécanisme de Galilée, Descartes, Harvey et Boyle. Bunge ne se contente pas de défendre le réalisme «mécaniste » mais critique sévèrement tout anti-réalisme: "Le programme phénoméniste chargé de rendre compte de la constance des successions d'une façon purement descriptive, sans rechercher les 'mécanismes' de ces changements, est en fait caractéristique d'un état de développement culturel relativement pauvre plutôt qu'un état d'avancement proprement 'positif' de l'humanité1 » (p. 49).

1. Un autre passage donne une idée de l'aspect pamphlétaire du livre de Bunge — qui n'est pas sans rappeler l'esprit dixhuitiémiste : "L'antiréalisme est déconnecté d'avec la science et la technologie, motivées par l'exploration et la transformation de la réalité. L'antiréalisme n'est pas seulement une erreur; il est complètement destructif par ce qu'il proclame être un vide total: ontologique, épistémologique, sémantique, méthodologique, axiologique, éthique et pratique. Un nihilisme ou un négativisme aussi intégral, faisant écho au bouddhisme, décourage non seulement l'évaluation objective et l'action rationnelle, mais aussi l'exploration du monde. C'est au mieux un divertissement académique» (p. 87). 
Bunge développe son épistémologie à partir de ce qu'il estime être la pratique scientifique, soit la découverte de mécanismes. Un mécanisme est un processus (une séquence d'état) dans un système concret, qu'il soit social ou naturel (p. 124). La notion de système descend en droite ligne du matérialisme systémique du baron d'Holbach selon lequel, en plus d'être matériel, le monde constitue un ensemble de systèmes diversement reliés. On caractérise habituellement le «système " comme un objet complexe dont les parties ou les composants sont liés entre eux par certains genres de liens (logique ou matériel) (p. 126). La conceptualisation, ou modélisation des systèmes se fait typiquement en précisant : 1) l'ensemble des parties qui le compose ; 2) l'ensemble des facteurs environnementaux qui affectent le système ; 3) la structure ou les types de liens qui unissent les parties du système; et finalement, 4) les mécanismes (processus) qui font que le système se comporte de façon spécifique.

L'auteur affirme que cette approche systémique est celle utilisée par les scientifiques de toutes obédiences, tant en sciences naturelles qu'en sciences humaines. Ainsi entendue, l'approche systémique n'est pas une théorie particulière en philosophie des sciences mais plutôt une perspective ou une stratégie (ou encore, une heuristique) permettant de créer des projets de recherche devant mener à la compréhension du système étudié (p. 129). Pour l'épistémologie systémique, le mécanisme essentiel est une notion centrale; expliquer revient donc principalement à fournir un mécanisme essentiel. Plus précisément, les chercheurs sont, majoritairement, intéressés à comprendre une certaine fonction d'un système (la référentialité permise par le langage, l'oxygénation par le système cardiovasculaire, la distribution des richesses par le système économique, etc.). Dans ce cadre, fournir un mécanisme éclairant la fonction spécifique d'un système, c'est pourvoir un mécanisme essentiel. Par exemple, la capacité d'enregistrer des données est la fonction spécifique du système mnémonique du cerveau, le comprendre se fait par l'intermédiaire d'un mécanisme essentiel, en l'occurrence le mécanisme de potentiation à long terme.

Une dernière remarque concernant le mécanisme de Bunge : celui-ci souligne, à l'instar des autres mécanistes contemporains, que la possibilité même de décrire des régularités, à l'aide de lois, est permise par l'existence de ces mécanismes. Donc, les explications subsomptives (particulièrement le modèle $\mathrm{D}-\mathrm{N}$ ) sont des explications dérivées ou secondes par rapport aux explications mécanistes (et systémistes). Cette conséquence épistémologique montre bien l'engagement ontologique du réalisme; le monde est composé d'objets qui forment des systèmes, expliquer ne consiste donc pas à simplement formaliser des régularités mais plutôt à rendre compte de l'existence même de ces régularités (en montrant quelles sont leurs fonctions et comment les mécanismes génèrent ces fonctions).

Pour tout projet réellement ambitieux, il est difficile de ne pas osciller entre l'admiration et la condamnation pour démesure. D'abord, le livre de Bunge attaque à peu près tous les sujets et d'innombrables auteurs - à peu près tous s'étant fourvoyés. Ensuite, rares sont les discussions engagées avec les auteurs contemporains qui partagent globalement les vues de l'auteur, et il s'en trouve un bon nombre (les "mécanistes» constituent un courant inévitable en philosophie des sciences). L'impression laissée est celle d'un système du monde nouvelle version qui fait figure de proue dans un univers philosophique peuplé d'idéalistes et de constructivistes, où le seul espoir est de suivre l'auteur dans son retour aux sciences telles qu'elles se conçoivent et se pratiquent. Par exemple, on ne retrouve rien en ce qui concerne le débat épistémologique évoluant autour de la définition même de la connaissance 


\section{$612 \cdot$ Philosophiques / Automne 2008}

(naturalisme et normativisme) ni aucune mention des différentes versions du mécanisme qui ont été développées depuis les vingt dernières années. En outre, pour ce qui est de l'ontologie des propriétés, on ne retrouvera aucun parallèle concernant les dispositions (propriétés = dispositions ?) et la façon dont nous pouvons les connaître.

Bref, Chasing Reality (2007) est un orphelin: 1) l'argumentation de Bunge dérape souvent, face aux sympathisants idéalistes et constructivistes, en accusations vindicatives (note 1 ) et ne constitue donc pas un dialogue «à tête reposée » chargé de faire avancer le débat; 2) ceux qui sont globalement matérialistes et mécanistes (systémistes ou non) ne trouveront rien de neuf dans cet ouvrage. En plus d'être essentiellement constitué des mêmes éléments que les livres précédents, surtout Emergence and Convergence (2003), Chasing Reality nous donne l'impression que Bunge a résolu, en principe, toutes les grandes questions philosophiques et qu'il nous laisse, au menu des tâches philosophiques futures, un vague mandat de philosophie "pratique » (morale et politique) (p. 281).

DAVY MOUGENOT

UQAM

\section{Volker Peckhaus (Hrsg.) Oskar Becker und die Philosophie der Mathematik, München, Wilhelm Fink Verlag, 2005, 352 pages.}

Oskar Becker (1889-1957) a été mathématicien avant de devenir un philosophe influencé, d'abord par Husserl, et ensuite par Heidegger (au grand dam de Husserl). Ses deux ouvrages principaux sont Mathematische Existenz (1927) et Grundlagen der Mathematik in geschichtlicher Entwicklung (1954), où il s'inspire de Schelling et de la philosophie de l'existence de Heidegger pour tenter de justifier l'existence d'ordinaux transfinis dans la théorie axiomatique des ensembles. Il a aussi contribué à l'histoire des mathématiques et à la logique modale, entre autres : ce qui a été appelé le postulat de Becker stipule que le statut des modalités est toujours régi par la nécessité, par exemple si A est possible, A est nécessairement possible. Ses prises de position politiques lui ont valu la désaffection du milieu intellectuel après la dernière guerre mondiale.

Le présent ouvrage, qui fait suite à une première parution consacrée aux rapports entre la philosophie et les sciences chez Oskar Becker (Die Philosophie und die Wissenschaften, 2002), est un recueil de quinze travaux publié sous la direction du réputé philosophe et historien de la logique, Volker Peckhaus. Peckhaus y va de deux contributions critiques dans ce volume, l'une consacrée à l'interprétation beckerienne de la notion formaliste d'existence chez Hilbert, et l'autre au rapport entre Becker et son collègue de Freiburg, le mathématicien Ernst Zermelo.

Peckhaus critique Becker à juste titre pour ne pas avoir pris la juste mesure du concept d'existence dans la métamathématique de Hilbert, une méprise philosophique qui est sans doute due à son parti pris réaliste. Pour appuyer sa thèse de la neutralité ontologique du concept d'existence chez Hilbert, Peckhaus cite la correspondance de Cantor et Hilbert, en 1897, sur l'existence de la totalité des alephs ou des cardinaux transfinis (taw ( $\eta$ ou encore la totalité des omégas ou ordinaux transfinis (notée $\Omega$ ). Cantor est conscient qu'il ne s'agit pas là d'un ensemble complété ou bien défini. 